\title{
INTERSTELLAR CIRCULAR POLARIZATION OF UPPER SCORPIUS STARS
}

\author{
JA MES C. KEMP* \\ Institute for Astronomy, University of Hawaii, Honolulu, H.I. 96822, U.S.A.
}

\begin{abstract}
Circular polarization of interstellar or perhaps circumstellar origin has been clearly established in two reddened stars in the Upper Scorpius region, $o$-Sco and $\sigma$-Sco A; and detected preliminarily in a third, HD 154445 . In the Corning 5-60 filter band $\lambda \lambda 3800-4600$, measured values of $10^{4} q$ were: $o$-Sco, $-(2.9 \pm 0.2) ; \sigma$-Sco A, $-(1.7 \pm 0.2)$; and HD 154445, $+(2.8 \pm 0.8)$. Complete wavelength dependences $q(\lambda)$ for $o$-Sco and $\sigma$-Sco A are under study, but so far are known to have the following similarity. Each shows a broad maximum (of negative $q$ ) centered in the range $\lambda \lambda 3800-4300$; and the values are smaller by a factor $\sim 4$, but still negative, in the range $\lambda \lambda 5000-6000$. The effect here is most likely due to a twisting grain alignment along the line of sight. In that case a simple model predicts $q(\lambda) \propto\left(n^{\prime}{ }_{l}-n_{r}^{\prime}\right) \cdot\left(n^{\prime \prime}{ }_{l}-n^{\prime \prime}{ }_{r}\right) \theta_{0} z_{0}{ }^{2}$, where $n=n^{\prime}+i n^{\prime \prime}$ is a formal, complex refractive index (in van de Hulst's forward-scattering approximation), $l$ and $r$ referring to longitudinal and transverse grain axes, and $\theta_{0}$ is the total twist angle over the interaction length $z_{0}$. An alternate mechanism would be multiple scattering (at large angles, not along the line of sight) within asymmetrical, well-localized clouds around the individual stars. The similar sign and magnitudes of $q$ for $o$-Sco and $\sigma$-Sco A, which are $1.5^{\circ}$ apart in the sky, argue for the line-of-sight mechanism - for example through a large cloud in front of the entire local region. Comparative studies of many stars in Upper Scorpius will be of obvious interest.
\end{abstract}

\section{Observations and Results}

The theoretical description of interstellar absorption by van de Hulst predicts, or at least permits, the existence of circular as well as linear polarization. The most basic mechanism is that due to a twist in the alignment direction of particles along the line of sight. I report here detection of circular polarization in three reddened stars, which very likely can be attributed to this mechanism. On the first of the three, a separate report is in press (Kemp, 1972).

The stars are $o$-Sco, $\sigma$-Sco A, and HD 154445, A- and B-type stars in the ScorpioCentaurus association (Garrison, 1967). Measurements on $o$-Sco were made first on the 88-in. telescope at Mauna Kea Observatory. In view of the special significance of this finding I repeated the observations on another telescope (24-in. at Mauna Kea) to help rule out all possibility of spurious linear-circular effects in the telescope. In reality the ratio of linear to circular polarizations here is quite within manageable limits for our polarimetric system including the telescopes we use (Kemp et al., 1972). In so far as LCC effects in the polarimeter as such are concerned, in all these measurements I applied both of our procedures (modulator and base rotations) to guard against spurious results. In the 5-60 filter band the LCC term $\Delta q$ that had to be cancelled by such rotations was no more than a third of the measured $q$ for any of the three stars. There was no contamination from sky background, as checked in each case by sampling for circularly polarized flux in the adjacent sky.

* On leave from Dept. of Physics, University of Oregon, Eugene, Ore. 97403. 


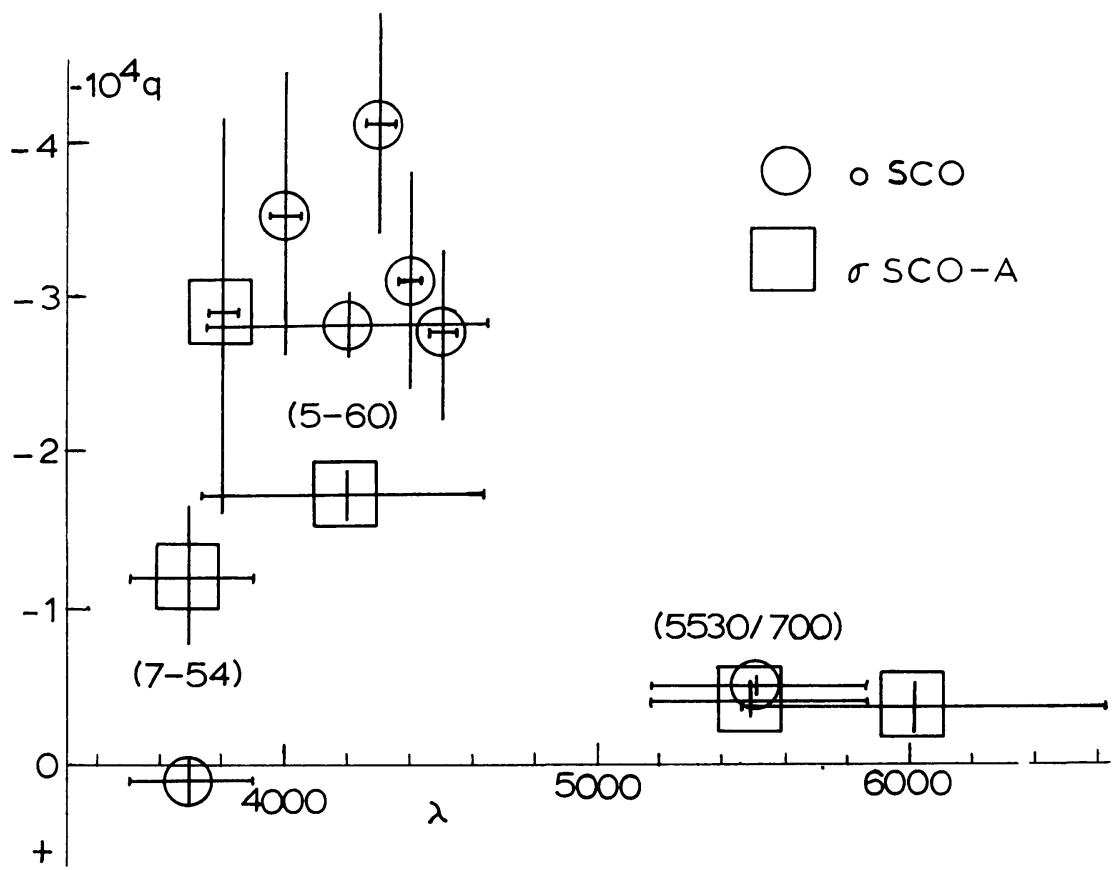

Fig. 1. Circular polarization data for $o$-Sco and $\sigma$-Sco A.Horizontal bars indicate the half-transmission widths of filters.

The fractional circular polarization values $q$ for these stars are shown in Figure 1 and Table I, variously for certain filter bands. My usual axial-vector sign convention is used: Positive $q$ means that if a fixed plane is placed across the line of sight between us and the star, we see the $\boldsymbol{E}$ vector as rotating counterclockwise in that plane. The $o$-Sco data are generally those taken as mentioned on the 88-in. Statistics there were adequate to allow use of some narrow-band $(100 \AA)$ filters. These were used to probe the region $\lambda \lambda 4000-4400$, of special interest for interstellar effects; of the three spectral regions examined so far this one showed the largest $q$. Best signal-to-noise obtained in a band $\lambda \lambda 3800-4600$ (Corning 5-60 filter). Measurements at the 24-in. on $o$-Sco were made with that filter, with results identical to those obtained on the 88-in. one month previous, to within $(\Delta q) / q \cong \pm 15 \%$ statistical and calibration error. Meas-

TABLE I

Data for three stars, circular polarization $q$ measured in $\lambda \lambda 3800-4600$ filter band

\begin{tabular}{llllllc}
\hline Star & $\begin{array}{l}\text { Spect. } \\
\text { type }\end{array}$ & $m_{V}$ & $E_{B-V}$ & $\begin{array}{l}10^{2} p \\
\text { (vis.) }\end{array}$ & $10^{4} q$ & $\begin{array}{l}\tilde{\theta}_{0} \\
\text { (radian) }\end{array}$ \\
\hline $\begin{array}{l}o \text {-Sco } \\
\text { HD 147084 }\end{array}$ & A5 & 4.6 & 0.70 & 4.4 & $-(2.9 \pm 0.2)$ & +0.4 \\
$\begin{array}{l}\sigma \text {-Sco A } \\
\text { HD 147165 }\end{array}$ & B1 & 2.9 & 0.40 & 1.6 & $-(1.7 \pm 0.2)$ & +2.0 \\
HD 154445 & B1 & 5.6 & 0.51 & 3.6 & $+(2.8 \pm 0.8)$ & -0.6 \\
\hline
\end{tabular}


urements at the 24-in. on the brighter star $\sigma$-Sco A were then made, in the filter bands shown in Figure 1; and, with long integration time in the 5-60 band but with adequate statistics in the result, on HD 154445. For comparison, an upper limit $q=-(0.05 \pm$ $0.10) \times 10^{-4}$ was recorded for $\alpha$-Sco (Antares); and a result with poor statistics, $q=+(0.3 \pm 0.2) \times 10^{-4}$, was obtained for $\delta$-Sco, both in the 5-60 band.

Results in the broad near UV band (Corning 7-54 filter) were possibly contaminated by the far-red leak in that filter around $\lambda$ 7200. In $o$-Sco this was not properly accounted for in Kemp (1972) and the small positive $q$ in that band is suspect, especially in view of the large reddening of the star. In $\sigma$-Sco A it was ascertained that the flux from the leak was only $10 \%$ of the total flux through the filter and the 7-54 result is probably reliable in this case. (The question is whether a large $q$, perhaps of positive sign, might obtain at $\lambda \gtrsim 7200$.)

The wavelength dependence is as yet fragmentary. I expect shortly to investigate one or more of the stars using filter widths of 100 to $200 \AA$ throughout $\lambda \lambda 3700-8500$, at the 88 -in. There is a crude indication (Figure 1) that $q(\lambda)$ for $o$-Sco and $\sigma$-Sco A may be similar.

\section{Discussion}

Using the effective refractive-index formalism of van de Hulst (1957) for line-of-sight propagation, if the alignment angle $\theta(z)$ (assuming elongated particles lying for simplicity in the $x y$ plane normal to the line of sight - Figure 3 ) is assumed slowly varying, it is easy to construct a pair of coupled differential equations for the $\boldsymbol{E}$-vector components $E_{x}, E_{y}$ governing the propagation. (Apparently equivalent equations in the Stokes-vector components are displayed in Serkowski (1962).) The problem here is that of a general gyrotropic medium and no doubt has been exhaustively studied, though I did not have a convenient reference at hand. For the case in which 0 varies linearly from zero (or strictly from some arbitrary initial direction) to a small angle $\theta_{0} \ll 1$ over the entire length $z_{0}$ of interaction with the particles, I solved these equations by a power-series expansion of $E_{x}(z), E_{y}(z)$. If the inital light is completely unpolarized, the resulting circular polarization is then:

$$
q \cong \frac{1}{3}\left(\frac{2 \pi}{\lambda}\right)^{2}\left(n_{l}^{\prime}-n_{r}^{\prime}\right)\left(n_{l}^{\prime \prime}-n_{r}^{\prime \prime}\right) \theta_{0} z_{0}^{2},
$$

where $\left(n_{l}^{\prime}, n_{l}^{\prime \prime}\right)$ are the real and imaginary parts respectively of the formal refractive index $n_{l}=n_{l}^{\prime}+i n_{l}^{\prime \prime}$ corresponding to the parallel axis of the grains, while $\left(n_{r}^{\prime}, n_{r}^{\prime \prime}\right)$ pertain to a perpendicular axis. In the same model the linear polarization is:

$$
p=\frac{2 \pi}{\lambda}\left(n_{l}^{\prime \prime}-n_{r}^{\prime \prime}\right) z_{0}
$$

so that we have the relationship:

$$
\theta_{0}=\left(\frac{3 q}{p^{2}}\right)\left[\frac{n_{l}^{\prime \prime}-n_{r}^{\prime \prime}}{n_{l}^{\prime}-n_{r}^{\prime}}\right] .
$$




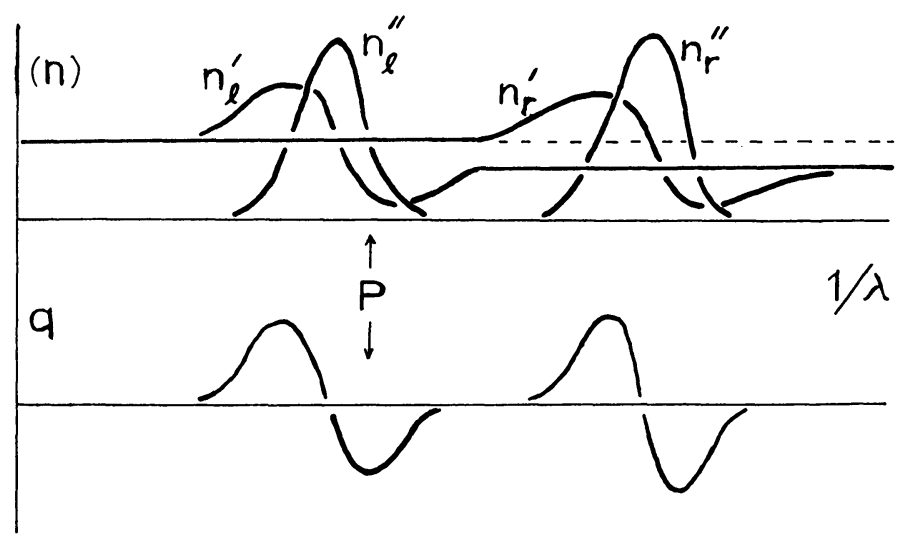

Fig. 2. Model for parallel and perpendicular refractive indices of hypothetical elongated or anisotropic aligned particles; and $q(\lambda)$ predicted by simple relationships in text.

It is reasonable to assume, on various general considerations including the implications of the Kramers-Kronig relations, that a peak value of $q$ corresponds to a wavelenght such that the dichroic-birefringent ratio in brackets above is of the order of unity. On the strength of a quite naive model for $n_{l, r}(\lambda)$ discussed below (Figure 2 etc.), we make the tentative guess that in our case for $\lambda \sim 4200$ this ratio is in fact negative, of order -1 . We therefore define an angle $\tilde{\theta}_{0} \equiv-\left(3 q / p^{2}\right)$, and list these values in Table I. This gives a rough measure of the total alignment rotation. If the sign of the dichroic-birefringent ratio has been guessed correctly, the signs of the $\hat{\theta}_{0}$ are the real ones; positive $\theta_{0}$ means that the alignment pattern along $z$ looks like a righthand screw. We note that the $\tilde{\theta}_{0}$ found here (Table I) are of the order of a radian, and are not really $\ll 1$ as assumed in deriving the above simple relations. Finding more exact formulae should not be difficult.

What we have here is a probe into the third dimensional aspect of interstellar polarization, thus perhaps of the magnetic alignment fields in space. In the present data, one question is whether the circular polarization is indeed due to the line-of-sight mecha-

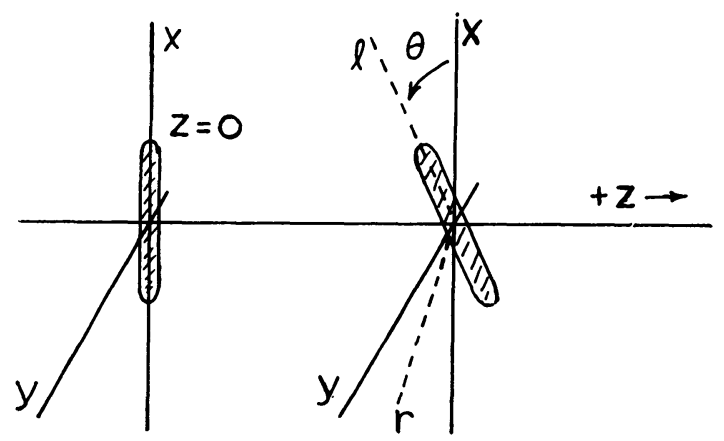

Fig. 3. Geometry for varying grain alignment along line of sight. Axes $l$ and $r$ lie in the transverse $x y$ plane. 
nism; or alternatively to highly localized asymmetric clouds or dust shells around (or adjacent to) individual stars, which can produce elliptical polarization by reflective effects, i.e. large-angle double scattering. It would seem unlikely that two stars ( $o$-Sco and $\sigma$-Sco A), nearby in space, could be chance have similarly disposed, asymmetric dust shells so similar in structure as to produce the observed $q$ values, which have the same sign and approximate magnitudes; this argues convincingly for the line-of-sight mechanism. The question then is whether we are seeing a diffuse galactic absorption, with the indicated variation of grain alignment occuring over a long path; or instead a somewhat localized cloud in front of or encompassing these stars in the upper Scorpius region. Observation of the interstellar $q$ for more stars in this region should bring the answer.

Finally we ask what light is shed by the circular polarization results on the chemistry or morphology of the interstellar grains. In Figure 2 we show the effective birefringence and dichroism (in the sense of the forward-scattering formalism of van de Hulst) of aligned grains of some unspecified character. We assume that the parallel and perpendicular excitations $(l, r)$ have predominant extinctions, respectively, defined by the two peaks $n_{l}^{\prime \prime}$ and $n_{r}^{\prime \prime}$ shown; these have corresponding real refractive indices $n_{l}^{\prime}$ and $n_{r}^{\prime}$. Based on the formula above, the resulting $q(1 / \lambda)$ is also displayed: It resembles the spectral derivative of the extinction peaks. Since the linear polarization $(p(\lambda)$ supposedly has the shape of the dichroism $\left(n_{l}^{\prime \prime}-n_{r}^{\prime \prime}\right)$, let us suppose that the typical maximum of $p$ around $\lambda 5000$ (from the work of Gehrels and others) corresponds to the broad peak of $n_{l}^{\prime \prime}$. The implication is that the peak in $q$ around $\lambda 4300$ is in the region $P$ of Figure 2; and we expect to find a reversed sign of $q$ in the near infrared. The prediction will be checked in the near future.

\section{Acknowledgements}

This work was by support to Mauna Kea Observatory by NASA, and the NSF.

\section{References}

Garrison, R. F.: 1967, Astrophys. J. 147, 1003.

Kemp, J. C.: 1972, Astrophys. J. 175, 35.

Kemp, J. C., Wolstencroft, R. D., and Swedlund, J. B.: 1972, Astrophys. J. $177,177$.

Serkowski, K.: 1962, Adv. Astron. Astrophys. 1, 289.

Van de Hulst, H. C.: 1957, Light Scattering by Small Particles, Wiley, New York. 